\title{
Determinants of Bicyclist Injury Severity Resulting from Crashes at Roundabouts, Crossroads, and T-Junctions
}

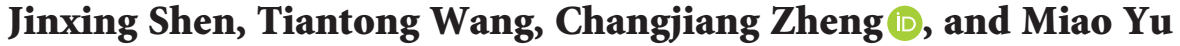 \\ College of Civil Engineering and Transportation Engineering, Hohai University, Nanjing 210098, China \\ Correspondence should be addressed to Changjiang Zheng; zheng@hhu.edu.cn
}

Received 26 February 2020; Revised 11 May 2020; Accepted 22 May 2020; Published 10 June 2020

Academic Editor: Feng Chen

Copyright (C) 2020 Jinxing Shen et al. This is an open access article distributed under the Creative Commons Attribution License, which permits unrestricted use, distribution, and reproduction in any medium, provided the original work is properly cited.

\begin{abstract}
This study explores the contributing factors that influence bicyclist injury severity at three types of intersection: roundabouts, crossroads, and T-junctions. Using bicycle-involved crash data in the UK over nine years (from 2009 to 2017), the bicyclist injury severity (with three severity levels: fatal injury, serious injury, and slight injury) was estimated using the generalized ordered logit (GOL) model and partial proportional odds (PPO) model. The marginal effects of each explanatory variable were computed to investigate the impacts on bicyclist injury severity occurring probabilities. A wide range of variables potentially affecting injury severity was considered, including bicyclist characteristics, intersection characteristics, environmental conditions, bicyclist movement and location preceding the crash, and types of collisions. Our findings show that the PPO model outperforms the GOL model for analyzing the factors that affect the bicyclist injury severity at intersections. The factors that affect cycling safety at various intersections show enormous differences. Specifically, nine variables have significant impacts on bicyclist injury severity at those three types of intersections. And there are only two variables, four variables, and eleven variables that have significant impact on bicyclist injury severity at roundabouts, crossroads, and T-junctions, respectively. The findings of this study can help decision makers better understand the spatial heterogeneity of the factors that influence the bicyclist injury severity at various intersections.
\end{abstract}

\section{Introduction}

Cycling is often considered as an economical, convenient, healthy, and sustainable transportation mode, especially suitable for short distance travel, which can offer a wide range of environmental and social benefits [1]. Recently, with the implementation of more than 20,000 bike-sharing schemes around the world, cycling has become a conventional travel mode in many cities [2]. With this in mind, the UK government has designed a series of policies over the past decade to promote the use of the bicycle in the daily journey, with the ambition to increase the bicycle trips from 0.8 billion in 2013 to 1.6 billion in 2025 [3]. Although the government has invested extensively to increase bikeability, bicycle travel mode only shares about $2 \%$ of all trips made in the UK, which is much lower than the Netherlands, Denmark, and Germany [4]. Among all the possible explanations, the most widely accepted view is that the safety risks perceived by cyclists are the most critical reasons hindering the increase of cycling share rate [5-10]. Therefore, it is significant to analyze and determine the influencing factors that affect the safety of bicycle trips, and thereby the government can develop the countermeasures accordingly to lower the severity of cycling risk and increase the level of bicycle use.

A series of studies have been conducted to examine the critical factors related to bicycle safety, including the influence of bicyclist and driver demographics, bicycle and vehicle characteristics, road and environmental factors, and other variables. Behnood and Mannering [11] identified the contributing factors of race, gender, age, and whether the bicyclist wears a helmet that can significantly affect the severity of the bicycle crash. The cyclists who are younger, less educated, and ride longer per week were associated with a higher safety risk [12]. Besides, since bicyclists aged over 65 need more time to perceive and respond to external information, they are more prone to be involved in severe bicycle crashes [13]. By analyzing the police-reported data, 
Kim et al. [14] implied that inclement weather, darkness without lighting, speeding, and involvement of trucks could significantly increase the probability of fatal bicyclist injury, and the fault of bicyclists is more likely to result in severe crashes than the drivers. Specifically, Eluru et al. [15] asserted that the age of bicyclists, the speed limit strategies of the road, and the locations and the period of crashes occurrence are the critical factors impacting the bicyclist injury severity. Cycling on the curved road segments, rural roads, and high-speed roads could raise the risk of severe injury [16]. Also, intoxicated bicyclists and automobile drivers, vans, SUVs, light-duty trucks, and roads with a grade or a curve are more likely to be involved in severe crashes [17]. Regarding the bicycle lanes, Morrison et al. [18] insisted that setting up exclusive bicycle lanes was a solution to improve the safety of bicyclists, which reduces the crash risk between bicycles and vehicles and raises the perception of cycling safety, thereby attracting more people to use bikes. However, the level of traffic pressure influenced the outcomes of bicycle lanes on cycling safety, and bicycle lanes on the roads with heavy traffic were more prone to involved in bicycle crashes [19]. Furthermore, the configuration of the adjacent intersections, bicycle traffic volume, and traffic control strategies at intersections could influence the effectiveness of bicycle lanes [20]. Besides, Klassen et al. [21] indicated that the essential factors influencing the severity of bicycle crash at intersections and road segments were not the same. Thus, unique treatments were needed to improve cycling safety at these two types of locations.

Several studies have verified that intersections are particularly dangerous areas due to the crossing traffic streams. Bicyclist crashes at intersections could increase the probability of severe injuries and fatalities [22-26]. Moore et al. [27] stated that there were essential differences in some factors that impact the bicyclist injury severity at intersections and non-intersections, and it was necessary to develop separate models to assess the effects of various factors on the bicyclist injury severity, respectively. Wang et al. [28] believed that the implementation of traffic calming methods, improving street lighting, and stop control strategy could enhance the cycling safety at nonsignalized intersections. Moreover, providing warning information to right-turning drivers when they approach the intersections can reduce conflicts between right-turning vehicles and bicycles on bicycle lanes, which can effectively enhance cycling safety at intersections [29]. Wang and Akar [30] concluded that the provision of bicycle boxes, bicycle crossing signs, and crossing markings at intersections could improve cycling safety, and the safety perceptions varied depending on the typologies of bicyclists. To sum up, although increasing researchers are starting to explore bicyclist safety at intersections, to the best of our knowledge, there is few detailed analysis for the difference of factors affecting bicyclist injury severity at various intersections.

Given the above, the objective of this research is to analyze and compare the influences of different intersection features on bicyclist injury severities in crashes. The cycling crash data used in this study are police-reported, occurred at various intersections in the UK, and the statistical period is nine years from January 1, 2009, to December 31, 2017. Specifically, we primarily focus on studying the factors affecting bicyclist injury severity cycling crashes at roundabouts, crossroads, and T-junctions. The principal reason is that the probability of bicycle crashes occurring at these three intersections were higher than others in the UK, according to bicycle crash data. The remainder of the paper is organized as follows. Section 2 summarizes and describes the methodology applied to analyze bicyclist injury severity as well as the methods for comparing the models. Section 3 presents data on bicycle crashes that occurred at roundabouts, crossroads, and T-junctions. Section 4 discusses the outcomes of the model estimation and marginal effects, and finally, a conclusion is given in Section 5.

\section{Methodologies}

In police-reported bicycle crashes, the bicyclist severity is generally recorded using ordinal categories, and it is classified as fatal injury, serious injury, and slight injury. The ordered logit model is used to analyze bicycle crashes, which needs to obey the parallel lines or proportional odds (PO) assumption, and the estimated parameters are the same across the cumulative level $[31,32]$. However, some variables affecting the bicycle crash levels at various intersections may be different. Following the recent studies such as Marcoux et al. [33], the generalized ordered logit model (GOL), which can relax the PO assumption for all variables, is selected in this study. Actually, in this study, we are not convinced whether we need to relax the PO constraint for all or some specific variables. Rationally, the partial proportional odds model (PPO) is also selected, for which only partial variables can violate the PO assumption. Moreover, using the same bicycle crash data, comparative analysis of the GOL and PPO model is conducted in this study. The brief information about the two models (GOL and PPO) is described as follows.

2.1. Generalized Ordered Logit (GOL) Model. In this paper, bicyclist injuries are studied by three discrete severity levels according to the police-reported bicycle crashes; accordingly, we coded $1=$ slight injury, 2 = serious injury, and 3 =fatal injury. And the contribution of driver characteristics, driver behaviors, collision types, infrastructure characteristics, vehicle types, and environmental conditions is assessed by the crash injury severity model. Following the research by Williams [34], we define the bicyclist injury severity function $y_{i}^{*}$ based on the latent regression:

$$
y_{i}^{*}=X_{i} \beta_{j}+\varepsilon_{i}
$$

where $j$ are the categories of bicyclist injury severities, $X_{i}$ is a $1 \times p$ vector that contains the values of all the explanatory variables to the bicycle crash $i, \beta_{j}$ is a vector of regression coefficients, $\varepsilon_{i}$ a residual term following a logistic distribution, and $y_{i}^{*}$ is a latent preference variable. The observed counterpart to $y_{i}^{*}$ is $y_{i}$, and the severity level $y_{i}$ of crash $i$ is defined as follows: 


$$
y_{i}= \begin{cases}1, & y_{i}^{*} \leq \mu_{i, 0} \\ 2, & \mu_{i, 0}<y_{i}^{*} \leq \mu_{i, 1} \\ 3, & \mu_{i, 1}<y_{i}^{*}\end{cases}
$$

where $\mu_{i, 0}, \mu_{i, 1}$, and $\mu_{i, 2}$ are the boundaries between the bicyclist severity levels for crash $i$. As the residual term $\varepsilon_{i}$ follows the logistic distribution, the GOL model can be written as

$$
P\left(y_{i}>j\right)=g\left(X_{i} \beta_{j}\right)=\frac{\exp \left(\alpha_{j}+X_{i} \beta_{j}\right)}{1+\exp \left(\alpha_{j}+X_{i} \beta_{j}\right)},
$$

where $\alpha_{j}$ represents a cutoff point for the cumulative logit of category $j$. From the above, it can be determined that the probabilities that $y_{i}$ will take on each of the values 1,2 , and 3 are equal to

$$
\left\{\begin{array}{l}
P\left(y_{i}=1\right)=1-g\left(X_{i} \beta_{1}\right) \\
P\left(y_{i}=2\right)=g\left(X_{i} \beta_{2}\right)-g\left(X_{i} \beta_{1}\right), \\
P\left(y_{i}=3\right)=g\left(X_{i} \beta_{2}\right) .
\end{array}\right.
$$

2.2. Partial Proportional Odds (PPO) Model. As mentioned above, unlike the GOL model, the PPO model allows some independent variables to violate the PO assumption, and other independent variables can remain constant for each crash injury severity level. Based on equation 3, suppose that there are only $m$ variables that obey the PO assumption, and the GOL model can be written as

$$
\begin{aligned}
P\left(y_{i}>j\right) & =g\left(X_{i, m} \beta+X_{i, p-m} \beta_{j}\right) \\
& =\frac{\exp \left(\alpha_{j}+X_{i, m} \beta+X_{i, p-m} \beta_{j}\right)}{1+\exp \left(\alpha_{j}+X_{i, m} \beta+X_{i, p-m} \beta_{j}\right)},
\end{aligned}
$$

where $X_{i, m}$ is a vector of $m$ explanatory variables to the bicycle crash $i$ that satisfies the PO assumption, $\beta$ is a vector of regression coefficients that is the same for all values of $j, X_{i, p-m}$ is a vector of $p-m$ variables to the bicycle crash $i$ that is free to the PO assumption, and $\beta_{j}$ is a vector of regression coefficients that is different for various values of $j$.

By conducting the Brant test for all independent variables, we can determine variables that satisfy the PO assumption. Particularly, when the independent variables pass the Brant test, it can be considered that those variables satisfy the PO assumption; otherwise, those variables need to be relaxed. For a detailed discussion on this, please refer to Williams [34].

2.3. Model Comparison. In this study, we use the same dataset to fit the GOL model and the PPO model and choose the log-likelihood of the full model $\left(L L_{f}\right)$, Akaike Information Criterion (AIC), and Bayesian Information Criterion (BIC) to compare the performance of those two models.

In the previous study, researchers have proven that AIC and BIC are practical evaluation criteria to assess the quality of different statistical models $[35,36]$. By comprehensively considering the penalty term of the number of predictive variables and the log-likelihood value of those two models, AIC and BIC consider not only the effect of model fitting but also the model complexity. The smaller the values of AIC and $\mathrm{BIC}$ are, the better the model fit effect is. The AIC and BIC can be calculated as follows:

$$
\begin{aligned}
& \mathrm{AIC}=2 k-2 L L_{f}, \\
& \mathrm{BIC}=k \ln (O)-2 L L_{f},
\end{aligned}
$$

where $k$ is the number of parameters estimated in the model and $O$ is the number of observations.

\section{Data Description}

The data used in this study were obtained from policereported cycling crashes that occurred at various intersections in the UK during the nine years from January 1, 2009, to December 31, 2017. According to the latest census, the total population of the UK is about 63.2 million, making it one of the most densely populated areas in the world.

The data used in the study were all obtained from the British Government Digital Service (https://data.gov.uk/ dataset/). In the UK, intersections are grouped into five categories in bicycle crash dataset reported by the police, including roundabouts, crossroads, T-junction, more than four arms but not a roundabout, and others. From 2009 to 2018, there are about 44,804 police-reported bicyclist injuries that occurred at various intersections in the UK (with all incomplete or incorrect data observations removed), and about $95 \%$ of which happened at or near roundabouts, intersections, and T-junction intersections, as shown in Table 1.

According to the statistical characteristics of bicycle crash data at various intersections, we primarily analyze bicyclist injury severity at three categories, including T-junction, roundabout, and crossroads. The characteristics defined in the dataset, including bicyclist characteristics, intersection attributes, environmental factors, bicyclist movement and location factors, and crash characteristics, are studied in this research for their effect on bicyclist injury severity.

The final analysis dataset contains 42,532 crashes, and the descriptive statistics of the variables used are shown in Table 2.

\section{Results and Discussion}

In this study, the GOL model and PPO model are fitted by a user-written program gologit 2 in Stata 15, and the coefficients of the explanatory variables in these two models were estimated by the maximum likelihood estimation. Estimation results of the GOL and PPO models are shown in Tables 3 and 4, respectively. The summaries of indicators for model comparison are given in Table 5. It is worth noting that the variables excluded in the final model are those that are not statistically significant, at least at the 95\% level ( $p$ value smaller than 0.05 ). 
Table 1: The statistics of total bicycle crashes at various intersections (2009-2018).

\begin{tabular}{lccccc}
\hline Intersection categories & T-junction & Roundabout & Crossroads & Others & Summary \\
\hline Total number & 26475 & 9127 & 6931 & 2272 & 44804 \\
Percentage (\%) & 59.09 & 20.37 & 15.47 & 5.07 & 100 \\
\hline
\end{tabular}

TABle 2: Descriptive statistics of the variables used in the estimations.

\begin{tabular}{|c|c|c|c|c|c|c|}
\hline \multirow{2}{*}{ Variable } & \multicolumn{2}{|c|}{ Roundabouts } & \multicolumn{2}{|c|}{ Crossroads } & \multicolumn{2}{|c|}{ T-junctions } \\
\hline & Mean & S.D. & Mean & S.D. & Mean & S.D. \\
\hline \multicolumn{7}{|l|}{ Bicyclist characteristics } \\
\hline Male ( 1 if bicyclist is male; 0 others) & 0.809 & 0.394 & 0.792 & 0.406 & 0.803 & 0.397 \\
\hline Age 1 ( 1 if bicyclist is younger than 15 years; 0 others) & 0.040 & 0.195 & 0.09 & 0.286 & 0.115 & 0.319 \\
\hline Age 2 ( 1 if bicyclist is older than 16 years and is younger than 25 years; 0 others) & 0.172 & 0.377 & 0.209 & 0.406 & 0.197 & 0.397 \\
\hline Age 3 ( 1 if bicyclist is older than 26 years and is younger than 35 years; 0 others) & 0.234 & 0.423 & 0.281 & 0.449 & 0.246 & 0.430 \\
\hline Age 4 ( 1 if bicyclist is older than 36 years and is younger than 45 years; 0 others) & 0.235 & 0.424 & 0.207 & 0.405 & 0.202 & 0.401 \\
\hline Age 5 ( 1 if bicyclist is older than 46 years and is younger than 55 years; 0 others) & 0.195 & 0.396 & 0.133 & 0.339 & 0.150 & 0.358 \\
\hline Age 6 ( 1 if bicyclist is older than 55 years; 0 others) & 0.125 & 0.330 & 0.081 & 0.272 & 0.090 & 0.286 \\
\hline Citizen ( 1 if bicyclist dwells in the city; 0 others) & 0.885 & 0.319 & 0.937 & 0.243 & 0.906 & 0.292 \\
\hline Towner ( 1 if bicyclist dwells in the town; 0 others) & 0.055 & 0.228 & 0.029 & 0.167 & 0.047 & 0.212 \\
\hline Villager ( 1 if bicyclist dwells in the rural area; 0 others) & 0.060 & 0.237 & 0.034 & 0.182 & 0.047 & 0.212 \\
\hline Part-of-work ( 1 if journey as part of work; 0 others) & 0.098 & 0.297 & 0.066 & 0.249 & 0.069 & 0.253 \\
\hline To-from-work ( 1 if comr & 0.215 & 0.411 & 0.176 & 0.381 & 0.164 & 0.370 \\
\hline Taking-pupil-school ( 1 if taking pupil to/from school; 0 others) & 0.014 & 0.118 & 0.010 & 0.100 & 0.013 & 0.114 \\
\hline Pupil-school (1 if pupil riding to/from schoo & 0.013 & 0.114 & 0.018 & 0.134 & 0.026 & 0.158 \\
\hline Purpose-others ( 1 if travel purpose is different from the above four models; 0 others) & 0.661 & 0.473 & 0.730 & 0.444 & 0.728 & 0.445 \\
\hline \multicolumn{7}{|l|}{ Intersection characteristics } \\
\hline Speed-limit (1 if the speed limit & 0.791 & 0.406 & & 0.253 & & 0.261 \\
\hline Junction-control ( 1 if the intersection is $n$ & 0.953 & 0.212 & 0.517 & 0.500 & 0.907 & 0.290 \\
\hline $\begin{array}{l}\text { Nonsignal-pedestrian ( } 1 \text { if pedestrian crossing facilities are nonsignalized controlled } \\
\text { crosswalks; } 0 \text { others) }\end{array}$ & 0.116 & 0.321 & 0.111 & 0.315 & 0.126 & 0.332 \\
\hline Signal-pedestrian ( 1 if there is pedestrian phase at traffic signal junction; 0 others) & 0 & 1 & 50 & 77 & 73 & 0.261 \\
\hline Footbridge ( 1 if there are footbridges or subways & 0.013 & 0.114 & 0.009 & 0.095 & 0.010 & 0.100 \\
\hline Central-refuge ( 1 if there are central refuges; 0 others) & 0.079 & 0.270 & 0.018 & 0.134 & 0.015 & 0.122 \\
\hline None ( 1 if there are no pedestrian crossing facilities; 0 others) & 0.791 & 0.406 & 0.931 & 0.253 & 0.927 & 0.261 \\
\hline Road-dry (1 if $\mathrm{r}$ & & & & 0.412 & 0.79 & 0.407 \\
\hline Road & 0.257 & 0. & 0.194 & 0.395 & 0.195 & 0.396 \\
\hline Road-ice ( 1 if roa & 0.011 & 0. & 0.014 & 0.118 & 0.007 & 0.084 \\
\hline Road-others ( 1 if road surface $\mathrm{i}$ & 0.007 & 0. & 0.010 & 0.100 & 0.008 & 0.089 \\
\hline Urban-junction (1 if the & 0.781 & & & 0.261 & & 0.318 \\
\hline Divider ( 1 if the roads hav & 0.167 & 0.373 & 0.260 & 0.438 & 0.264 & 0.440 \\
\hline \multicolumn{7}{|l|}{ Environmental condition } \\
\hline Weekend ( 1 if the crash o & 0.243 & 0.429 & 0.256 & 0.436 & 0.273 & 0.445 \\
\hline Morning-peak ( 1 if the crash occurred at the morning rush hour; 0 ot & 0.202 & 0.401 & 0.165 & 0.371 & 0.190 & 0.392 \\
\hline Night-peak ( 1 if the crash occurred at the afternoon rush hour; 0 others & 0.177 & 0.382 & 0.205 & 0.404 & 0.227 & 0.418 \\
\hline & & & & & & 0.493 \\
\hline Daylight ( 1 if it was daylight when the crash occurred; 0 others) & 0.754 & 0.430 & 0.761 & 0.427 & 0.850 & 0.358 \\
\hline Night-light ( 1 if the crash occurred at night, and there were lights on the road; 0 ot & 0.221 & 0.415 & 0.219 & 0.414 & 0.189 & 0.391 \\
\hline $\begin{array}{l}\text { Night-nonlight ( } 1 \text { if the crash occurred at night, and there were no lights on the road; } 0 \\
\text { others) }\end{array}$ & 0.02 & 0.158 & 0.020 & 41 & 0.039 & 0.192 \\
\hline Weather-fine ( 1 if it was fine weather when the crash occurred; 0 others) & 0.835 & 0.371 & 0.849 & 0.358 & 0.871 & 0.335 \\
\hline Weather-raining ( 1 if it was raining when the crash occur & 0.122 & 0.327 & 0.097 & 0.297 & 0.100 & 0.300 \\
\hline Weather-snowing ( 1 if it was snowing when the crash occl & 0.008 & 0.089 & 0.011 & 0.105 & 0.012 & 0.110 \\
\hline Weather-foggy ( 1 if it was foggy when the crash occurred; 0 others) & 0.010 & 0.100 & 0.011 & 0.105 & 0.009 & 0.095 \\
\hline Weather-others ( 1 if it was other weather when the crash occurred; 0 ot & 0.025 & 0.155 & 0.032 & 0.176 & 0.009 & 0.095 \\
\hline January ( 1 if the crash occurred in January; 0 others) & 0.089 & 0.285 & 0.066 & 0.249 & 0.071 & 0.257 \\
\hline February ( 1 if the crash occurred in February; 0 others) & 0.073 & 0.261 & 0.063 & 0.243 & 0.063 & 0.243 \\
\hline March ( 1 if the crash occurred in March; 0 others) & 0.072 & 0.259 & 0.080 & 0.272 & 0.080 & 0.272 \\
\hline April ( 1 if the crash occurred in April; 0 others) & 0.073 & 0.261 & 0.078 & 0.268 & 0.075 & 0.263 \\
\hline May ( 1 if the crash occurred in May; 0 others) & 0.082 & 0.274 & 0.09 & 0.286 & 0.092 & 0.290 \\
\hline June ( 1 if the crash occurred in June; 0 others) & 0.080 & 0.272 & 0.095 & 0.293 & 0.097 & 0.297 \\
\hline
\end{tabular}


TABLE 2: Continued.

\begin{tabular}{|c|c|c|c|c|c|c|}
\hline \multirow{2}{*}{ Variable } & \multicolumn{2}{|c|}{ Roundabouts } & \multicolumn{2}{|c|}{ Crossroads } & \multicolumn{2}{|c|}{ T-junctions } \\
\hline & Mean & S.D. & Mean & S.D. & Mean & S.D. \\
\hline July (1 if the crash occurred in July; 0 others) & 0.092 & 0.290 & 0.102 & 0.303 & 0.100 & 0.300 \\
\hline August ( 1 if the crash occurred in August; 0 others) & 0.081 & 0.272 & 0.089 & 0.285 & 0.085 & 0.279 \\
\hline September ( 1 if the crash occurred in September; 0 others) & 0.091 & 0.288 & 0.091 & 0.288 & 0.100 & 0.300 \\
\hline October ( 1 if the crash occurred in October; 0 others) & 0.099 & 0.298 & 0.101 & 0.302 & 0.096 & 0.295 \\
\hline November ( 1 if the crash occurred in November; 0 others) & 0.094 & 0.292 & 0.088 & 0.283 & 0.085 & 0.279 \\
\hline December ( 1 if the crash occurred in December; 0 others) & 0.075 & 0.263 & 0.057 & 0.232 & 0.057 & 0.232 \\
\hline \multicolumn{7}{|l|}{ Bicyclist movement and location preceding the crash } \\
\hline Parked ( 1 if the bicycle is in a state of parking; 0 otl & 0.007 & 0.084 & 0.01 & 0.100 & 0.013 & 0.114 \\
\hline Waiting-go ( 1 if the bike is waiting to go straight; 0 others) & 0.014 & 0.118 & 0.018 & 0.134 & 0.031 & 0.173 \\
\hline Slowing ( 1 if the bicycle is in a state of deceleration; 0 others) & 0.013 & 0.114 & 0.02 & 0.141 & 0.061 & 0.239 \\
\hline Moving-off ( 1 if the bicycle is moving off; 0 others) & 0.038 & 0.192 & 0.036 & 0.187 & 0.058 & 0.235 \\
\hline Lefting ( 1 if the bike is turning left; 0 others) & 0.007 & 0.084 & 0.010 & 0.100 & 0.010 & 0.100 \\
\hline Waiting-left ( 1 if the bike is waiting to turn left; 0 others) & 0.008 & 0.089 & 0.010 & 0.100 & 0.012 & 0.110 \\
\hline Righti & 0.124 & 0.330 & 0.051 & 0.219 & 0.064 & 0.245 \\
\hline Waiting-right & 0.008 & 0.089 & 0.025 & 0.155 & 0.042 & 0.200 \\
\hline Lane-left ( 1 if the bike is changing lane to left; & 0.009 & 0.095 & 0.010 & 0.100 & 0.014 & 0.118 \\
\hline Lane-right ( 1 if the & 0.012 & 0.110 & 0.015 & 0.122 & 0.017 & 0.130 \\
\hline Over-offside ( 1 if the bike is & 0.016 & 0.126 & 0.022 & 0.148 & 0.031 & 0.173 \\
\hline Over-nearside $(1$ & 0.011 & 0.105 & 0.030 & 0.170 & 0.027 & 0.161 \\
\hline Aheac & 0.013 & 0.114 & 0.014 & 0.118 & 0.011 & 0.105 \\
\hline Ahead-right ( & 0.056 & 0.230 & 0.017 & 0.130 & 0.026 & 0.158 \\
\hline Ahead ( 1 if the bike is goi & 0.665 & 0.472 & 0.715 & 0.452 & 0.582 & 0.493 \\
\hline Main & 0.954 & 0.210 & 0.927 & 0.261 & 0.894 & 0.308 \\
\hline on busway; 0 others) & 0.019 & 0.138 & 0.023 & 0.148 & 0.03 & 0.170 \\
\hline Cycleway ( 1 if the bike is on cycleway; 0 others) & 0.014 & 0.118 & 0.030 & 0.170 & 0.049 & 0.217 \\
\hline Pavemer & 0.007 & 0.0 & 0.011 & 0.105 & 0.020 & 0.141 \\
\hline ers ( 1 if the bike is o & 0.007 & 0.084 & 0.009 & 0.095 & 0.009 & 0.095 \\
\hline $\begin{array}{l}\text { Approaching-parked ( } 1 \text { if the bike is approaching junction or waiting/parked at junction } \\
\text { approach; } 0 \text { others) }\end{array}$ & 0.209 & 0.4 & 0.278 & 0.448 & 0.367 & 0.482 \\
\hline Leaving-parked ( 1 if bike is leaving junction or waiting/parked at junction exit; 0 others) & 0.156 & & 0.071 & 0.257 & 0.085 & 0.279 \\
\hline Leaving-m & 0.016 & 0.126 & 0.012 & 0.110 & 0.018 & 0.134 \\
\hline d; 0 others) & 0.016 & 0.126 & 0.049 & 0.217 & 0.054 & 0.226 \\
\hline Mid ( 1 if the bike is located in the in & 0.604 & 0.489 & 0.59 & 0.492 & 0.476 & 0.499 \\
\hline \multicolumn{7}{|l|}{ Type of collision } \\
\hline Collision-point-fr & 0.346 & 0.475 & 0.528 & 0.499 & 0.552 & 0.497 \\
\hline nt-back ( 1 if the collisio & 0.181 & 0.385 & 0.086 & 0.281 & 0.084 & 0.277 \\
\hline Collision-point-right ( 1 if the collision point is on the right side of the bike; 0 others) & 0.139 & 0.346 & 0.219 & 0.414 & 0.185 & 0.389 \\
\hline Collision-point-left ( 1 if the collision point is on the left side of the bike; 0 others) & 0.301 & 0.458 & 0.131 & 0.338 & 0.136 & 0.344 \\
\hline Collision-point-none ( 1 if there was no collision point; 0 others) & 0.034 & 0.182 & 0.037 & 0.190 & 0.044 & 0.205 \\
\hline Secondary-collision-on-road ( 1 if the secondary collision occurred & 0.011 & 0.105 & 0.026 & 0.158 & 0.032 & 0.176 \\
\hline Secondary-collision-off-road ( 1 if the secondary collision occurred o & 0.017 & 0.130 & 0.014 & 0.118 & 0.007 & 0.084 \\
\hline Secondary-collision-none ( 1 if there is no secondary collision occurred; 0 & 0.972 & 0.164 & 0.96 & 0.195 & 0.961 & 0.192 \\
\hline
\end{tabular}

4.1. Comparison of Models. In this study, according to the estimates of the GOL and PPO models, we adopt AIC, BIC, and pseudo $R^{2}$ to compare those two models (as shown in Table 5). It can be concluded that the AIC and BIC values of the PPO model are smaller than those of the GOL model. These two values imply that, given the same dataset, the PPO model produces better fitting results. Besides, a similar implication can be derived from the pseudo $R^{2}$ because the value of the PPO model is larger than that of the GLO model. In summary, the PPO model outperforms the GOL model for fitting the data of bicycle crashes that occurred at various intersections. We mainly adopt the PPO model to analyze the bicycle crash data.

It should be noted that the sign of the estimated coefficients cannot intuitively interpret the influence of explanatory variables on the PPO model outcomes. To present meaningful explanations, we calculate the marginal effects of each variable to evaluate the impacts of estimates on the bicyclist injury severity occurring probabilities. Particularly, the marginal effects demonstrate the difference in outcome probability of each level of bicyclist injury severity caused by one unit change in the explanatory variable (as shown in Table 6).

4.2. Bicyclist Characteristics. In Table 3, several factors are found to be statistically significant in influencing severity outcomes in bicyclist-related crashes. Specifically, it is found that male cyclists are more likely to be involved in fatal or serious injuries at crossroads and T-junctions while 
TABle 3: Estimation results of the GOL model.

\begin{tabular}{|c|c|c|c|c|c|c|}
\hline \multirow{2}{*}{ Variables } & \multicolumn{2}{|c|}{ Roundabouts } & \multicolumn{2}{|c|}{ Crossroads } & \multicolumn{2}{|c|}{ T-junctions } \\
\hline & Fatal & Serious & Fatal & Serious & Fatal & Serious \\
\hline \multicolumn{7}{|l|}{ Bicyclist characteristics } \\
\hline Male & & $0.100(0.052)^{*}$ & & $-0.169(0.063)^{* *}$ & & $-0.088(0.033)^{* *}$ \\
\hline Age 1 & & $0.804(0.132)^{* * *}$ & & $0.32(0.105)^{* *}$ & & $0.298(0.053)^{* * *}$ \\
\hline Age 2 & & $0.652(0.071)^{* * *}$ & & $0.389(0.084)^{* * *}$ & & $0.38(0.043)^{* * *}$ \\
\hline Age 3 & $1.46(0.585)^{*}$ & $0.461(0.062)^{* * *}$ & & $0.398(0.080)^{* * *}$ & & $0.324(0.041)^{* * *}$ \\
\hline Age 4 & & $0.330(0.060)^{* * *}$ & & $0.191(0.082)^{*}$ & & $0.154(0.041)^{* * *}$ \\
\hline Age 6 & & $-0.25(0.066)^{* * *}$ & $-1.306(0.495)^{* *}$ & $-0.305(0.096)^{* *}$ & $-1.501(0.280)^{*}$ & $-0.279(0.048)^{* * *}$ \\
\hline Villager & & & & & $-0.675(0.246)^{* *}$ & $-0.111(0.057)^{*}$ \\
\hline Part-of-work & & $0.202(0.074)^{* *}$ & & & & $0.15(0.052)^{* *}$ \\
\hline To-from-work & & & & & $1.012(0.370)^{* *}$ & \\
\hline Pupil-school & & & & & & $0.366(0.098)^{* * *}$ \\
\hline \multicolumn{7}{|l|}{$\begin{array}{l}\text { Intersection } \\
\text { characteristics }\end{array}$} \\
\hline Speed-limit & & $0.331(0.048)^{* * *}$ & $0.944(0.409)^{*}$ & $0.529(0.097)^{* * *}$ & $1.239(0.231)^{* * *}$ & $0.441(0.047)^{* * *}$ \\
\hline Junction-control & $1.613(0.437)^{* * *}$ & & $0.967(0.342)^{* *}$ & & $0.723(0.235)^{* *}$ & \\
\hline Divider & $-1.053(0.402)^{* *}$ & & & & $-0.958(0.394)^{*}$ & $-0.29(0.076)^{* * *}$ \\
\hline Urban-junction & & & & $0.278(0.099)^{* *}$ & $0.45(0.246)^{*}$ & $0.321(0.042)^{* * *}$ \\
\hline \multicolumn{7}{|l|}{$\begin{array}{l}\text { Environmental } \\
\text { condition }\end{array}$} \\
\hline Weather-fine & & & & $0.266(0.125)^{*}$ & & \\
\hline Weather-raining & & $0.188(0.066)^{* *}$ & & $0.291(0.146)^{*}$ & & \\
\hline Night-peak & & & & & & $0.071(0.032)^{*}$ \\
\hline Night-light & & & & & & $-0.122(0.033)^{* * *}$ \\
\hline Night-nonlight & & & & & & $-0.195(0.074)^{* *}$ \\
\hline February & & & & & $-0.701(0.266)^{* *}$ & \\
\hline May & & & & $-0.14(0.084)^{*}$ & & \\
\hline June & & $0.19(0.080)^{*}$ & & & & \\
\hline October & & & & $-0.142(0.079)^{*}$ & & \\
\hline \multicolumn{7}{|l|}{$\begin{array}{l}\text { Bicyclist movement } \\
\text { and location preceding } \\
\text { the crash }\end{array}$} \\
\hline Parked & & & & & $-1.971(1.068)^{*}$ & $1.354(0.600)^{*}$ \\
\hline Waiting-go & & & & $0.889(0.277)^{* *}$ & & \\
\hline Righting & & $-0.234(0.089)^{* *}$ & & & $-0.494(0.248)^{*}$ & $-0.113(0.051)^{*}$ \\
\hline Waiting-right & & & & $0.764(0.377)^{*}$ & & $0.418(0.122)^{* *}$ \\
\hline Ahead-right & & $-0.446(0.106)^{* * *}$ & & & & $-0.24(0.073)^{* *}$ \\
\hline Over-nearside & $-2.928(0.907)^{* *}$ & & & & $-1.241(0.404)^{* *}$ & \\
\hline Lane-left & & $-0.483(0.212)^{*}$ & & & & \\
\hline Approaching-parked & & & $1.523(0.606)^{*}$ & & & \\
\hline Leaving-parked & & & & & $-0.825(0.256)^{* *}$ & $-0.09(0.046)^{*}$ \\
\hline Entering-main & & & $-1.038(0.400)^{* *}$ & $-0.206(0.110)^{*}$ & $-1.149(0.281)^{* * *}$ & $-0.182(0.057)^{* *}$ \\
\hline Busway & & & & & $-1.387(0.768)^{*}$ & \\
\hline Pavement & & $-0.534(0.298)^{*}$ & & & $-1.35(0.615)^{*}$ & \\
\hline \multicolumn{7}{|l|}{ Type of collision } \\
\hline Collision-point-front & & & & $0.51(0.115)^{* * *}$ & & $0.422(0.055)^{* * *}$ \\
\hline Collision-point-back & & $0.234(0.057)^{* * *}$ & & $0.804(0.144)^{* * *}$ & & $0.652(0.070)^{* * *}$ \\
\hline Collision-point-right & & $0.15(0.063)^{*}$ & & $0.576(0.122)^{* * *}$ & & $0.444(0.060)^{* * *}$ \\
\hline Collision-point-left & & & & $0.416(0.128)^{* *}$ & & $0.544(0.063)^{* * *}$ \\
\hline $\begin{array}{l}\text { Secondary-collision-on- } \\
\text { road }\end{array}$ & & & & & & $-0.264(0.067)^{* * *}$ \\
\hline $\begin{array}{l}\text { Secondary-collision-off- } \\
\text { road }\end{array}$ & & $-0.78(0.248)^{* *}$ & & $-0.482(0.278)^{*}$ & $-1.491(0.405)^{* * *}$ & $-0.679(0.125)^{* * *}$ \\
\hline Constant & $4.394(0.784)$ & $0.957(0.132)$ & $2.746(1.325)$ & $-0.04(0.208)$ & $4.339(0.542)$ & $0.227(0.088)$ \\
\hline Number of observations & \multicolumn{2}{|c|}{16418} & \multicolumn{2}{|c|}{11,623} & \multicolumn{2}{|c|}{14491} \\
\hline $\begin{array}{l}\text { Log-likelihood at zero, } \\
L L(0)\end{array}$ & \multicolumn{2}{|c|}{-7800.35} & \multicolumn{2}{|c|}{-5544.79} & \multicolumn{2}{|c|}{-6810.67} \\
\hline $\begin{array}{l}\text { Log-likelihood at } \\
\text { convergence, } L L(\beta)\end{array}$ & \multicolumn{2}{|c|}{-6817.51} & \multicolumn{2}{|c|}{-4823.41} & \multicolumn{2}{|c|}{-5932.77} \\
\hline Pseudo $R^{2}$ & & 260 & & 301 & & 289 \\
\hline AIC & 15 & 4.71 & 111 & 39.57 & 136 & 52.38 \\
\hline BIC & 16 & 8.36 & 115 & 57.61 & 139 & 39.59 \\
\hline
\end{tabular}

Note. Standard errors are in parentheses. Level of significance: $*$ indicates parameter is significant at $0.05, * *$ indicates parameter is significant at 0.01 , and $* * *$ indicates parameter is significant at 0.001 . 
TABLE 4: Estimation results of the PPO model.

\begin{tabular}{|c|c|c|c|c|c|c|}
\hline \multirow{2}{*}{ Variables } & \multicolumn{2}{|c|}{ Roundabouts } & \multicolumn{2}{|c|}{ Crossroads } & \multicolumn{2}{|c|}{ T-junctions } \\
\hline & Fatal & Serious & Fatal & Serious & Fatal & Serious \\
\hline \multicolumn{7}{|l|}{ Bicyclist characteristics } \\
\hline Male & $0.111(0.052)^{*}$ & $0.111(0.052)^{*}$ & $-0.16(0.064)^{*}$ & $-0.16(0.064)^{*}$ & $-0.089(0.033)^{* *}$ & $-0.089(0.033)^{* *}$ \\
\hline Age 1 & $0.706(0.143)^{* * *}$ & $0.706(0.143)^{* * *}$ & $0.309(0.111)^{* * *}$ & $0.309(0.110)^{*}$ & $0.275(0.053)^{* * *}$ & $0.275(0.053)^{* * *}$ \\
\hline Age 2 & $0.654(0.071)^{* * *}$ & $0.654(0.071)^{* * *}$ & & $0.396(0.085)^{* * *}$ & $0.373(0.043)^{* * *}$ & $0.373(0.043)^{* * *}$ \\
\hline Age 3 & $0.467(0.062)^{* * *}$ & $0.467(0.062)^{* * *}$ & $0.405(0.080)^{* * *}$ & $0.405(0.080)^{* * *}$ & $0.325(0.041)^{* * *}$ & $0.325(0.041)^{* * *}$ \\
\hline Age 4 & $0.336(0.060)^{* * *}$ & $0.336(0.060)^{* * *}$ & $0.194(0.082)^{* *}$ & $0.194(0.082)^{* *}$ & $0.156(0.041)^{* * *}$ & $0.156(0.041)^{* * *}$ \\
\hline Age 6 & $-0.263(0.066)^{* * *}$ & $-0.263(0.066)^{* * *}$ & $-1.571(0.354)^{* * *}$ & $-0.298(0.097)^{* *}$ & $-1.426(0.178)^{* * *}$ & $-0.282(0.048)^{* * * *}$ \\
\hline Villager & & & & & $-0.716(0.235)^{* *}$ & $-0.114(0.057)^{*}$ \\
\hline Part-of-work & $0.188(0.075)^{*}$ & $0.188(0.075)^{*}$ & & & $0.146(0.052)^{* *}$ & $0.146(0.052)^{* *}$ \\
\hline To-from-Work & & & & & $0.981(0.366)^{* *}$ & \\
\hline Pupil-school & & & & & $0.366(0.098)^{* * *}$ & $0.366(0.098)^{* * *}$ \\
\hline \multicolumn{7}{|l|}{ Intersection characteristics } \\
\hline Speed-limit & $0.284(0.054)^{* * *}$ & $0.284(0.054)^{* * *}$ & $1.327(0.340)^{* * *}$ & $0.537(0.097)^{* * *}$ & $1.287(0.188)^{* * *}$ & $0.443(0.047)^{* * *}$ \\
\hline Junction-control & $1.492(0.424)^{* * *}$ & & $0.843(0.318)^{* * *}$ & & $0.711(0.232)^{* *}$ & \\
\hline Divider & $-1.095(0.397)^{* *}$ & & & & $-0.296(0.076)^{* * *}$ & $-0.296(0.076)^{* * *}$ \\
\hline Urban-junction & $0.115(0.055)^{*}$ & $0.115(0.055)^{*}$ & $0.255(0.110)^{* *}$ & $0.255(0.110)^{* *}$ & $0.329(0.041)^{* * *}$ & $0.329(0.041)^{* * *}$ \\
\hline Road-wet & $-4.438(1.873)^{*}$ & $-4.438(1.873)^{*}$ & & & & \\
\hline \multicolumn{7}{|l|}{ Environmental condition } \\
\hline Weather-fine & & & $0.313(0.131)^{* *}$ & $0.313(0.131)^{* *}$ & & \\
\hline Weather-raining & $0.193(0.067)^{* *}$ & $0.193(0.067)^{* *}$ & $0.308(0.161)^{* *}$ & $0.308(0.1$ & & \\
\hline Weather-foggy & & & $-2.202(1.064)^{* *}$ & & & \\
\hline Night-peak & & & & & $0.073(0.032)^{*}$ & $0.073(0.032)^{*}$ \\
\hline Night-lig & & & & & $-0.118(0.033)^{* * *}$ & $-0.118(0.033)^{* * *}$ \\
\hline Night-nonlight & & & & & $-0.193(0.074)^{* *}$ & $-0.193(0.074)^{* *}$ \\
\hline February & & & & & $-0.641(0.263)^{*}$ & \\
\hline May & & & $-0.192(0.105)^{* *}$ & $-0.192(0.105)^{* *}$ & & \\
\hline June & $0.208(0.108)^{*}$ & $0.208(0.108)^{*}$ & & & & \\
\hline October & & & $-0.179(0.101)^{* *}$ & $-0.179(0.101)^{* *}$ & & \\
\hline \multicolumn{7}{|l|}{$\begin{array}{l}\text { Bicyclist movement and } \\
\text { location preceding the crash }\end{array}$} \\
\hline Parked & & & & & $-2.288(1.003)^{*}$ & $1.314(0.600)^{*}$ \\
\hline Waiting-go & & & $0.869(0.292)^{* * *}$ & $0.869(0.292)^{* * *}$ & $0.801(0.172)^{* * *}$ & $0.801(0.172)^{* * *}$ \\
\hline Righting & & & & & $-0.115(0.050)^{*}$ & $-0.115(0.050)^{*}$ \\
\hline Waiting-right & & & $0.733(0.388)^{*}$ & $0.733(0.388)^{* *}$ & $0.4(0.122)^{* *}$ & $0.4(0.122)^{* *}$ \\
\hline Over-nearside & $-2.531(0.759)^{* *}$ & & $-2.294(0.466)^{* * *}$ & & $-1.212(0.393)^{* *}$ & \\
\hline Ahead-right & $-0.38(0.120)^{* *}$ & $-0.38(0.120)^{* *}$ & & & $-0.216(0.072)^{* *}$ & $-0.216(0.072)^{* *}$ \\
\hline Entering-main & & & $-2.57(1.063)^{* *}$ & & $-1.179(0.239)^{* * *}$ & $-0.233(0.055)^{* * *}$ \\
\hline Lane-left & $-0.441(0.220)^{*}$ & $-0.441(0.220)^{*}$ & $-1.034(0.561)^{*}$ & $-1.034(0.561)^{*}$ & & \\
\hline Busway & & & & & $-1.389(0.587)^{* *}$ & \\
\hline Pavement & $-0.495(0.242)^{*}$ & -0.4 & & & $-1.285(0.345)^{* * *}$ & \\
\hline \multicolumn{7}{|l|}{ Type of collision } \\
\hline Collision-point-front & & & $-0.511(0.116)^{* * *}$ & $-0.511(0.116)^{* * *}$ & $-0.446(0.055)^{* * *}$ & $-0.446(0.055)^{* * *}$ \\
\hline Collision-point-back & $0.407(0.117)^{* * *}$ & $0.407(0.117)^{* * *}$ & $0.812(0.146)^{* * *}$ & $0.812(0.146)^{* * *}$ & & $0.656(0.070)^{* * *}$ \\
\hline Collision-point-right & & $0.324(0.119)^{* *}$ & & $0.575(0.123)^{* * *}$ & & $0.457(0.060)^{* * *}$ \\
\hline Collision-point-left & & & & $0.414(0.130)^{* * *}$ & $0.576(0.063)^{* * *}$ & $0.576(0.063)^{* * *}$ \\
\hline Secondary-collision-on-road & & & & & $-0.319(0.066)^{* * *}$ & $-0.319(0.066)^{* * *}$ \\
\hline Secondary-collision-off-road & $-0.753(0.253)^{* *}$ & $-0.753(0.253)^{* *}$ & $-0.477(0.281)^{*}$ & $-0.477(0.281)^{*}$ & $-1.636(0.386)^{* * *}$ & $-0.69(0.125)^{* * *}$ \\
\hline Constant & $4.576(1.191)$ & $1.042(1.123)$ & $4.787(1.613)$ & $-0.849(1.460)$ & $4.000(0.301)$ & $0.528(0.087)$ \\
\hline Number of obser & \multicolumn{2}{|c|}{16418} & \multicolumn{2}{|c|}{11623} & \multicolumn{2}{|c|}{14491} \\
\hline Log-likelihood at zero, $L L(0)$ & \multicolumn{2}{|c|}{-7778.23} & \multicolumn{2}{|c|}{-5525.77} & \multicolumn{2}{|c|}{-6810.30} \\
\hline $\begin{array}{l}\text { Log-likelihood at } \\
\text { convergence, } L L(\beta)\end{array}$ & \multicolumn{2}{|c|}{-6776.39} & \multicolumn{2}{|c|}{-4788.63} & \multicolumn{2}{|c|}{-5929.73} \\
\hline Pseudo $R^{2}$ & 0.12 & 288 & 0.13 & & & 293 \\
\hline AIC & 1566 & 69.03 & & 6.97 & 1362 & 27.88 \\
\hline $\mathrm{BIC}$ & 1586 & 69.39 & 1142 & 4.59 & 1380 & 0.70 \\
\hline
\end{tabular}

Note. Standard errors are in parentheses. Level of significance: $*$ indicates parameter is significant at $0.05, * *$ indicates parameter is significant at 0.01 , and *** indicates parameter is significant at 0.001 .

having a decreased likelihood of a slight injury, consistent with previous research $[11,14,15]$. The average marginal effects (as shown in Table 5) show that at crossroads and
T-junctions, the male indicator variable increases the probability of serious injuries by $2.37 \%$ and $1.24 \%$, respectively. Instead, the variable increases the probability of 
TABle 5: Indicators for model comparison.

\begin{tabular}{lccccccccc}
\hline \multirow{2}{*}{ Model } & \multicolumn{3}{c}{ Roundabouts } & \multicolumn{3}{c}{ Crossroads } & \multicolumn{2}{c}{ T-junctions } \\
& AIC & BIC & Pseudo $R^{2}$ & AIC & BIC & Pseudo $R^{2}$ & AIC & BIC & Pseudo $R^{2}$ \\
\hline GOL & 15684.71 & 16008.36 & 0.1260 & 11189.57 & 11557.61 & 0.1301 & 13662.38 & 13989.59 & 0.1289 \\
PPO & 15669.03 & 15869.39 & 0.1288 & 11166.97 & 11424.59 & 0.1334 & 13627.88 & 13800.70 & 0.1293 \\
\hline
\end{tabular}

TABle 6: Marginal effects for different bicycle crash injury severity levels at different intersections.

\begin{tabular}{|c|c|c|c|c|c|c|c|c|c|}
\hline \multirow{2}{*}{ Variables } & \multicolumn{3}{|c|}{ Roundabouts } & \multicolumn{3}{|c|}{ Crossroads } & \multicolumn{3}{|c|}{ T-junctions } \\
\hline & Fatal & Serious & Slight & Fatal & Serious & Slight & Fatal & Serious & Slight \\
\hline \multicolumn{10}{|l|}{ Bicyclist characteristics } \\
\hline Male & -0.0003 & -0.0145 & 0.0148 & 0.0007 & 0.0237 & -0.0244 & 0.0003 & 0.0124 & -0.0127 \\
\hline Age 1 & -0.0022 & -0.1149 & 0.1171 & -0.0013 & -0.0448 & 0.0461 & -0.0009 & -0.0384 & 0.0392 \\
\hline Age 2 & -0.0018 & -0.0927 & 0.0945 & 0.0020 & -0.0584 & 0.0564 & -0.0012 & -0.0519 & 0.0531 \\
\hline Age 3 & -0.0012 & -0.0655 & 0.0667 & -0.0016 & -0.0562 & 0.0578 & -0.0010 & -0.0453 & 0.0463 \\
\hline Age 4 & -0.0009 & -0.0472 & 0.0481 & -0.0008 & -0.0269 & 0.0276 & -0.0005 & -0.0217 & 0.0222 \\
\hline Age 6 & 0.0007 & 0.0361 & -0.0368 & 0.0060 & 0.0372 & -0.0433 & 0.0045 & 0.0356 & -0.0402 \\
\hline Villager & & & & & & & 0.0023 & 0.0140 & -0.0163 \\
\hline Part-of-work & -0.0005 & -0.0288 & 0.0293 & & & & -0.0005 & -0.0203 & 0.0208 \\
\hline To-from-Work & & & & & & & -0.0031 & 0.0073 & -0.0042 \\
\hline Pupil-school & & & & & & & -0.0012 & -0.0510 & 0.0521 \\
\hline \multicolumn{10}{|l|}{ Intersection characteristics } \\
\hline Speed-limit & -0.0008 & -0.0405 & 0.0413 & -0.0054 & -0.0706 & 0.0760 & -0.0041 & -0.0590 & 0.0631 \\
\hline Junction-control & -0.0040 & -0.0121 & 0.0161 & -0.0034 & -0.0065 & 0.0100 & -0.0023 & -0.0081 & 0.0103 \\
\hline Divider & 0.0030 & 0.0034 & -0.0064 & & & & 0.0009 & 0.0412 & -0.0422 \\
\hline Urban-junction & -0.0003 & -0.0139 & 0.0142 & -0.0011 & -0.0394 & 0.0405 & -0.0010 & -0.0458 & 0.0469 \\
\hline Road-wet & 0.0115 & 0.6092 & -0.6207 & & & & & & \\
\hline \multicolumn{10}{|l|}{ Environmental condition } \\
\hline Weather-fine & & & & -0.0010 & -0.0369 & 0.0379 & & & \\
\hline Weather-raining & -0.0022 & -0.1179 & 0.1201 & -0.0011 & -0.0398 & 0.0409 & & & \\
\hline Weather-foggy & & & & 0.0086 & -0.0166 & 0.0080 & & & \\
\hline Night-peak & & & & & & & -0.0002 & -0.0102 & 0.0104 \\
\hline Night-light & & & & & & & 0.0004 & 0.0165 & -0.0169 \\
\hline Night-nonlight & & & & & & & 0.0006 & 0.0269 & -0.0275 \\
\hline February & & & & & & & 0.0020 & -0.0033 & 0.0013 \\
\hline May & & & & 0.0006 & 0.0199 & -0.0204 & & & \\
\hline June & -0.0005 & -0.0270 & 0.0275 & & & & & & \\
\hline October & & & & 0.0006 & 0.0203 & -0.0208 & & & \\
\hline \multicolumn{10}{|c|}{$\begin{array}{l}\text { Bicyclist movement and location preceding the } \\
\text { crash }\end{array}$} \\
\hline Parked & & & & & & & 0.0072 & -0.1945 & 0.1872 \\
\hline Waiting-go & & & & -0.0038 & -0.1343 & 0.1381 & -0.0025 & -0.1116 & 0.1142 \\
\hline Righting & & & & & & & 0.0004 & 0.0160 & -0.0163 \\
\hline Waiting-right & & & & -0.0033 & -0.1162 & 0.1195 & -0.0013 & -0.0558 & 0.0570 \\
\hline Over-nearside & 0.0065 & -0.0094 & 0.0029 & 0.0076 & -0.0101 & 0.0025 & 0.0038 & -0.0157 & 0.0119 \\
\hline Ahead-right & 0.0009 & 0.0455 & -0.0463 & & & & 0.0007 & 0.0301 & -0.0307 \\
\hline Entering-main & & & & 0.0054 & 0.0241 & -0.0295 & 0.0037 & 0.0295 & -0.0332 \\
\hline Lane-left & 0.0010 & 0.0504 & -0.0513 & 0.0036 & 0.1288 & -0.1324 & & & \\
\hline Busway & & & & & & & 0.0044 & -0.0059 & 0.0015 \\
\hline Pavement & 0.0014 & 0.0733 & -0.0747 & & & & 0.0041 & 0.0151 & -0.0192 \\
\hline \multicolumn{10}{|l|}{ Type of collision } \\
\hline Collision-point-front & & & & 0.0020 & 0.0696 & -0.0715 & 0.0014 & 0.0622 & -0.0636 \\
\hline Collision-point-back & -0.0007 & -0.0356 & 0.0363 & -0.0032 & -0.1140 & 0.1172 & -0.0002 & -0.0932 & 0.0934 \\
\hline Collision-point-right & 0.0016 & -0.0238 & 0.0222 & 0.0012 & -0.0832 & 0.0820 & 0.0009 & -0.0661 & 0.0651 \\
\hline Collision-point-left & & & & 0.0023 & -0.0608 & 0.0585 & -0.0018 & -0.0803 & 0.0821 \\
\hline Secondary-collision-on-road & & & & & & & 0.0010 & 0.0445 & -0.0455 \\
\hline Secondary-collision-off-road & 0.0021 & 0.1098 & -0.1119 & 0.0019 & 0.0680 & -0.0699 & 0.0052 & 0.0931 & -0.0983 \\
\hline
\end{tabular}


serious injuries that happened at the roundabout by $1.45 \%$. The primary reason for this phenomenon may be that bicyclists ordinarily consider that traffic conflicts at roundabouts are more severe, and they could be more careful, which results in lower serious accidents [27].

The age of bicyclists is also a statistically significant variable to analyze the injury severity. Particularly, older bicyclists (older than 55 years) are more likely to be involved in the occurrence of serious injury. According to the average marginal effects in Table 6, this variable increases the occurrence probability of serious injury at roundabouts, crossroads, and $\mathrm{T}$-junctions by $3.61 \%, 3.72 \%$, and $3.56 \%$, respectively. The result can be supported by previous research $[11,13,14,27]$. The possible reason for this finding is that the older bicyclists are slower and have a longer reaction and perception times than other age groups. For the younger bicyclists (the bicyclist is younger than 15 years), they are always involved in slight injuries. The indicator increases the probability of slight injury at roundabouts, crossroads, and T-junctions by $11.71 \%, 4.61 \%$, and $3.92 \%$, respectively. Besides, bicyclists who live in rural areas are more likely to be seriously injured in cycling crashes at T-junctions, and the variable increases the occurrence probability of slight injury by $1.4 \%$.

4.3. Intersection Characteristics. Regarding the intersection characteristics, in Table 5, many statistically significant factors influence bicyclist injury severity. Consistent with previous research [15], traffic control strategies enforced at intersections appear to be effective in reducing the possibility of serious and fatal injury. For instance, regarding the speed limit strategy at intersections, the factor decreases the occurrence probability of serious injuries that occurred at roundabouts, crossroads, and T-junctions by $4.05 \%, 7.06 \%$, and $5.9 \%$, respectively. However, nonsignalized control strategies at intersections decrease serious and fatal injury while increasing the possibility of slight injuries. Such factor increases the occurrence probability of slight injury at roundabouts, crossroads, and $\mathrm{T}$-junctions by $1.61 \%, 1 \%$, and $1.03 \%$, respectively. At the nonsignalized intersections, bicyclists and drivers might consciously slow down, thus effectively reducing the occurrence of fatal and severe casualties [28]. Similarly, bicyclists are less likely to be involved in fatal or severe crashes that occurred at urban intersections due to integrated traffic control stratagems.

The widely accepted view is that the implication of road facilities (for example, divider facilities between motor and bicycle lanes) can improve the safety of cyclists. However, this study finds that the provision of divider facilities at roundabouts and T-junctions increases the likelihood of fatal and serious injury. The indicator increases the occurrence probability of serious injury at $\mathrm{T}$-junctions by $4.12 \%$. It is mainly due to the fact that divider facilities are more likely to cause a secondary collision when the crash occurred, and the secondary collision will significantly increase the occurrence of fatal or severe casualties. It is worth noting that, consistent with the results in Kim et al. [14], cycling on wet roads was more prone to severe or fatal injury. To be specific, the indicator increases the occurrence probability of serious injuries that happened at the roundabouts by $60.92 \%$.

4.4. Environmental Conditions. Several environmental conditions related variables are found to affect bicyclist injury severity significantly, as shown in Table 5 . Consistent with previous research, bad weather can result in more dangerous cycling $[17,19,23]$. However, the impact of environmental conditions on the probability of cycling crashes that occurred at the various intersections is quite dissimilar. Interestingly, rainy days only affected the possibility of cycling crashes at roundabouts and intersections, and the variable decreases the likelihood of fatal and serious injury while increasing the occurrence probability of slight injury. In particular, on rainy days, more consideration should be paid to cycling safety at roundabouts, since the factor can increase the occurrence probability of slight injury at roundabouts by $12.01 \%$. Besides, foggy days only have a significant impact on cycling safety at crossroads. In particular, it significantly increases the likelihood of serious injury crashes, and the factor can increase the occurrence probability of serious injury by $12.01 \%$.

It is generally considered that lighting conditions are directly related to the visibility of the bicyclist and drivers, which will directly affect the severity of cycling crashes. However, in this study, we find out that lighting conditions only have a significant effect on the likelihood of serious cycling crashes at $\mathrm{T}$-junctions. Opening up the street lights at night reduces the possibility of the slight injury, and the lack of street lights increases the likelihood of serious injury. However, with or without street lights, we need to be more careful when cycling near T-junctions at night $[13,14]$.

In previous studies, researchers have found that seasons and months have a significant influence on the probability of bicyclist injury severity [27]. Furthermore, in this study, we discover February and June significantly affect bicyclist injury severity only at $\mathrm{T}$-junctions and roundabouts, separately. In particular, in May and October, we need to pay more attention to the possibility of severe cycling crashes at crossroads, and the factors increase the occurrence probability of serious injury by $1.99 \%$ and $2.03 \%$, respectively.

\subsection{Bicyclist Movement and Location Preceding the Crash.} In Table 5, a wide range of movement-related variables shows a statistically significant influence on the outcome of the bicyclist injury severity. Individually, at roundabouts, making a right turn, changing lanes to the left, and overtaking inside the intersections are statistically significant factors related to bicyclist injury severity. As for cross sections, stopped following going straight, stopped following turning right, overtaking inside the intersections, entering the main road from the intersection, and changing lanes to the left are statistically significant factors related to bicyclist injury severity. And the variables, including parked, stopped following going straight, making a right turn, waiting and turn right, overtaking inside the intersections, entering the main road from the intersection, and riding on the wrong road, will affect the severity of the injury that occurred at the T-junctions. 
Particularly, consistent with the previous research [11], parked or making a right turn has a significant effect on cycling safety. Interestingly, in this study, these two factors only appear to have statistically significant impact on the bicyclist injury severity at T-junctions, and the factor of parked at $\mathrm{T}$-junctions increases the occurrence probability of slight injury by $18.72 \%$ while decreasing the occurrence probability of serious injury by $19.45 \%$. However, changing lanes to the left does not show any significant influence on the likelihood of bicyclist injury severity at $\mathrm{T}$-junctions and shows a considerable effect on severe or fatal cycling crashes at roundabouts and cross sections. Correspondingly, the factor will increase the occurrence probability of serious injury at roundabouts and crossroads by $5.04 \%$ and $12.88 \%$, respectively. Moreover, cycling on the pavement can also significantly impact the likelihood of bicyclist injury severity at roundabouts and crossroads, and the factor increases the occurrence probability of serious injury that occurred at these two types of intersections by $7.33 \%$ and $1.51 \%$, respectively.

4.6. Type of Collision. As shown in Table5, the type-of-collision indicators, including the collision point at the front, back, right, and left, and the secondary collisions that occurred in or off the road, could impact the cycling crash injury severity, and the impact is statistically significant. Similar to previous studies $[13,14,27]$, this study also found that the severity of cycling crashes at various intersections can be distinctly affected by the collision point. Specifically, the collision point at the front and left merely appears to have statistically significant impact on the bicyclist injury severity at crossroads and T-junctions, mainly since there are few left-turn traffic volumes at roundabouts. The factor of the collision point at the front will increase the occurrence probability of serious injury at crossroads and $\mathrm{T}$-junctions by $6.96 \%$ and $6.22 \%$, respectively. In comparison, the factor of the collision point at the left will decrease the occurrence probability of serious injury at crossroads and $\mathrm{T}$-junctions by $6.08 \%$ and $8.03 \%$, respectively. Interestingly, in this study, we discover that the factors of the collision point at the back and left will decrease the probability of serious injury whiling increasing the likelihood of slight injury. Above all, the collision point at the front or back is mainly caused by cycling when going straight. Due to the faster speed of vehicles, serious cycling crash severity is more likely to occur. Besides, since the vehicle speed is always slower while turning at the intersections, the probability of slight injury is more likely to occur at the left and right collision points.

The widely accepted opinion is that the secondary collision is dangerous. However, in this study, we find that the indicator of secondary-collision-on-road only affects the severity of cycling crashes at T-junctions, and the factor increases the occurrence probability of serious injury by $4.45 \%$ while decreasing the occurrence probability of slight injury by $4.55 \%$. Also, it should be noted that the indicator of the secondary-collision-off-road significantly increases the likelihood of severe cycling crashes. In Table 6, the factor increases the occurrence probability of serious injury at roundabouts, crossroads, and T-junctions by $10.98 \%, 6.8 \%$, and $9.31 \%$, respectively.

\section{Conclusions}

To improve the cycling safety of bicyclists at various intersections, in this study, we apply the GOL model and PPO model to explore the possible factors that may result in the severity of bicycle injuries. Particularly, according to the statistical characteristics of data on bicycle crashes that occurred in the UK from 2009 to 2019, the intersections in this study are divided into three groups, including roundabouts, intersections, and T-shaped intersections. The bicyclist injury severity is divided into three categories: slight injury, serious injury, and fatal injury. A wide range of possible factors affecting bicyclist injury severities, including bicyclist characteristics, intersection characteristics, environmental conditions, bicyclist movement and location preceding the crash, and types of collisions, is considered.

The model estimation results reveal that the PPO model outperforms the GOL model for analyzing the factors that affect the severity of cycling crashes at various intersections. Further, we calculated the marginal effects of the variables in the PPO model to explore the differences of factors that influence the occurrence probability of bicyclist injury severity at various intersections. Regarding the estimation results of the bicyclist injury severity, we find out that there are gigantic differences in the factors that influence the severity of cycling crashes at various intersections. Particularly, we find that nine variables have significant impacts on bicyclist injury severity at those three types of intersections, including male bicyclists, age, speed limit, traffic control strategies at intersections, urban junctions, overtaking inside the intersections, the collision point at the back of the bicycle, the collision point at the right of the bike, and the secondary collision happened on the roadside. Interestingly, there are two variables (cycling in wet road and cycling in June) that only have significant impact on bicyclist injury severity at roundabouts. And four variables (cycling in find days, cycling in foggy days, cycling in May, and cycling in October) are discovered to only have significant impact on bicyclist injury severity at crossroads. Surprisingly, up to eleven variables are discovered to only have significant impact on bicyclist injury severity at $\mathrm{T}$-junctions, and the variables include the cyclist is a villager, journey purpose is to or from work, pupil is going to or from school alone, cycling in night peak hours, cycling in the night with a light on the road, cycling in the night without a light on the road, cycling in February, parked, turning right, cycling in the busway, and the secondary collision happened on the road.

Moreover, in this study, we also found that the factors affecting the cycling safety of intersections may also appear to have significant impact in different seasons, which means these factors may not be homogeneous with the time change. Due to the limitations of the PPO model, we cannot consider the temporal heterogeneity of various influencing factors in the analysis, and we will continue to focus on such issues in subsequent studies. With the growing importance relating to bicyclist safety, this paper provides some essential initial findings with the dataset from the UK and also provides some guidance for the analysis of cycling crashes from other countries. Anyway, this study can help decision makers 
better understand the spatial heterogeneity of the factors that influence the bicyclist injury severity at various intersections. Thus, more specific and efficient measures can be provided to enhance cycling safety at different types of intersections.

\section{Data Availability}

The data used in the study were all obtained from the British Government Digital Service (https://data.gov.uk/dataset/).

\section{Conflicts of Interest}

The authors declare that they have no conflicts of interest.

\section{Acknowledgments}

This research was supported by the National Natural Science Foundation of China (51808187), the Natural Science Foundation of Jiangsu Province (BK20170879), the Fundamental Research Funds for the Central Universities (2019B13514), and the Jiangsu Planned Projects for Postdoctoral Research Funds (1701086B).

\section{References}

[1] Q. Cai, J. Lee, N. Eluru, and M. Abdel-Aty, "Macro-level pedestrian and bicycle crash analysis: incorporating spatial spillover effects in dual state count models," Accident Analysis \& Prevention, vol. 93, pp. 14-22, 2016.

[2] Y. Ji, X. Ma, M. He, Y. Jin, and Y. Yuan, "Comparison of usage regularity and its determinants between docked and dockless bike-sharing systems: a case study in Nanjing, China," Journal of Cleaner Production, vol. 255, pp. 120110-120121, 2020.

[3] R. Cervero, S. Denman, and Y. Jin, "Network design, built and natural environments, and bicycle commuting: evidence from British cities and towns," Transport Policy, vol. 74, pp. 153164, 2019.

[4] N. Grudgings, A. Hagen-Zanker, S. Hughes, B. Gatersleben, M. Woodall, and W. Bryans, "Why don't more women cycle? An analysis of female and male commuter cycling mode-share in England and Wales," Journal of Transport \& Health, vol. 10, pp. 272-283, 2018.

[5] J. Lee, M. Abdel-Aty, Q. Cai, L. Wang, and H. Huang, "Integrated modeling approach for non-motorized mode trips and fatal crashes in the framework of transportation safety planning," Transportation Research Record: Journal of the Transportation Research Board, vol. 2672, no. 32, pp. 49-60, 2018.

[6] F. Feng, S. Bao, R. C. Hampshire, and M. Delp, "Drivers overtaking bicyclists-an examination using naturalistic driving data," Accident Analysis \& Prevention, vol. 115, pp. 98-109, 2018.

[7] R. Aldred, R. Goel, J. Woodcock, and A. Goodman, "Contextualising Safety in numbers: a longitudinal investigation into change in cycling safety in Britain, 1991-2001 and 2001-2011," Injury Prevention, vol. 25, no. 3, pp. 236-241, 2019.

[8] C. Ma, D. Yang, J. Zhou, Z. Feng, and Q. Yuan, "Risk riding behaviors of urban E-bikes: a literature review," International Journal of Environmental Research and Public Health, vol. 16, no. 13, pp. 1-18, 2019.

[9] L. Hou, J. Duan, W. Wang, R. Li, G. Li, and B. Cheng, "Drivers' braking behaviors in different motion patterns of vehicle-bicycle conflicts," Journal of Advanced Transportation, vol. 2019, Article ID 4023970, 17 pages, 2019.

[10] C. Ma, J. Zhou, D. Yang et al., "Research on the relationship between the individual characteristics of electric bike riders and illegal speeding behavior: a questionnaire-based study," Sustainability, vol. 12, no. 3, pp. 1-13, 2020.

[11] A. Behnood and F. Mannering, "Determinants of bicyclist injury severities in bicycle-vehicle crashes: a random parameters approach with heterogeneity in means and variances," Analytic Methods in Accident Research, vol. 16, pp. 35-47, 2017.

[12] X. Wu, W. Xiao, C. Deng, D. C. Schwebel, and G. Hu, "Unsafe riding behaviors of shared-bicycle riders in urban China: a retrospective survey," Accident Analysis \& Prevention, vol. 131, pp. 1-7, 2019.

[13] X. Yan, M. Ma, H. Huang, M. Abdel-Aty, and C. Wu, "Motor vehicle-bicycle crashes in Beijing: irregular maneuvers, crash patterns, and injury severity," Accident Analysis \& Prevention, vol. 43, no. 5, pp. 1751-1758, 2011.

[14] J.-K. Kim, S. Kim, G. F. Ulfarsson, and L. A. Porrello, "Bicyclist injury severities in bicycle-motor vehicle accidents," Accident Analysis \& Prevention, vol. 39, no. 2, pp. 238-251, 2007.

[15] N. Eluru, C. R. Bhat, and D. A. Hensher, "A mixed generalized ordered response model for examining pedestrian and bicyclist injury severity level in traffic crashes," Accident Analysis \& Prevention, vol. 40, no. 3, pp. 1033-1054, 2008.

[16] S. Boufous, L. de Rome, T. Senserrick, and R. Ivers, "Risk factors for severe injury in cyclists involved in traffic crashes in Victoria, Australia," Accident Analysis \& Prevention, vol. 49, pp. 404-409, 2012.

[17] E. Robartes and T. D. Chen, "The effect of crash characteristics on cyclist injuries: an analysis of Virginia automobile-bicycle crash data," Accident Analysis \& Prevention, vol. 104, pp. 165-173, 2017.

[18] C. N. Morrison, J. Thompson, M. C. Kondo, and B. Beck, "Onroad bicycle lane types, roadway characteristics, and risks for bicycle crashes," Accident Analysis \& Prevention, vol. 123, pp. 123-131, 2019.

[19] C. Chen, J. C. Anderson, H. Wang, Y. Wang, R. Vogt, and S. Hernandez, "How bicycle level of traffic stress correlate with reported cyclist accidents injury severities: a geospatial and mixed logit analysis," Accident Analysis \& Prevention, vol. 108, pp. 234-244, 2017.

[20] M. C. Kondo, C. Morrison, E. Guerra, E. J. Kaufman, and D. J. Wiebe, "Where do bike lanes work best? A bayesian spatial model of bicycle lanes and bicycle crashes," Safety Science, vol. 103, pp. 225-233, 2018.

[21] J. Klassen, K. El-Basyouny, and M. T. Islam, "Analyzing the severity of bicycle-motor vehicle collision using spatial mixed logit models: a City of Edmonton case study," Safety Science, vol. 62, pp. 295-304, 2014.

[22] J. Yuan and M. Abdel-Aty, "Approach-level real-time crash risk analysis for signalized intersections," Accident Analysis \& Prevention, vol. 119, pp. 274-289, 2018.

[23] Y. Wang and N. L. Nihan, "Estimating the risk of collisions between bicycles and motor vehicles at signalized intersections," Accident Analysis \& Prevention, vol. 36, no. 3, pp. 313-321, 2004.

[24] Y. Guo, Z. Li, Y. Wu, and C. Xu, "Exploring unobserved heterogeneity in bicyclists' red-light running behaviors at different crossing facilities," Accident Analysis \& Prevention, vol. 115, pp. 118-127, 2018.

[25] Z. Cheng, Z. Zu, and J. Lu, "Traffic crash evolution characteristic analysis and spatiotemporal hotspot identification of 
urban road intersections," Sustainability, vol. 11, no. 1, pp. 1-17, 2019.

[26] B. Yu, S. Bao, Y. Chen, and Y. Chen, "Using 3D mobile mapping to evaluate intersection design through drivers' visual perception," IEEE Access, vol. 7, pp. 19222-19231, 2019.

[27] D. N. Moore, W. H. Schneider, P. T. Savolainen, and M. Farzaneh, "Mixed logit analysis of bicyclist injury severity resulting from motor vehicle crashes at intersection and nonintersection locations," Accident Analysis \& Prevention, vol. 43, no. 3, pp. 621-630, 2011.

[28] C. Wang, L. Lu, and J. Lu, "Statistical analysis of bicyclists' injury severity at unsignalized intersections," Traffic Injury Prevention, vol. 16, no. 5, pp. 507-512, 2015.

[29] Y. Wu, M. Abdel-Aty, O. Zheng, Q. Cai, and L. Yue, "Developing a crash warning system for the bike lane area at intersections with connected vehicle technology," Transportation Research Record: Journal of the Transportation Research Board, vol. 2673, no. 4, pp. 47-58, 2019.

[30] K. Wang and G. Akar, "The perceptions of bicycling intersection safety by four types of bicyclists," Transportation Research Part F: Traffic Psychology and Behaviour, vol. 59, pp. 67-80, 2018.

[31] Q. Zeng, W. Hao, J. Lee et al., "Investigating the impacts of real-time weather conditions on freeway crash severity: a bayesian spatial analysis," International Journal of Environmental Research and Public Health, vol. 17, no. 8, pp. 1-15, 2020.

[32] F. Chen, M. Song, and X. Ma, "Investigation on the injury severity of drivers in rear-end collisions between cars using a random parameters bivariate ordered probit model," International Journal of Environmental Research and Public Health, vol. 16, no. 14, pp. 1-12, 2019.

[33] R. Marcoux, S. Yasmin, N. Eluru, and M. Rahman, "Evaluating temporal variability of exogenous variable impacts over 25 years: an application of scaled generalized ordered logit model for driver injury severity," Analytic Methods in Accident Research, vol. 20, pp. 15-29, 2018.

[34] R. Williams, "Understanding and interpreting generalized ordered logit models," The Journal of Mathematical Sociology, vol. 40, no. 1, pp. 7-20, 2016.

[35] F. Chen, S. Chen, and X. Ma, "Analysis of hourly crash likelihood using unbalanced panel data mixed logit model and real-time driving environmental big data," Journal of Safety Research, vol. 65, pp. 153-159, 2018.

[36] B. Dong, X. Ma, F. Chen, and S. Chen, "Investigating the differences of single-vehicle and multivehicle accident probability using mixed logit model," Journal of Advanced Transportation, vol. 2018, Article ID 2702360, 6 pages, 2018. 